\title{
Æolian tones and stall flutter of lengthy objects in fluid flows
}

\author{
P.S. Landa \\ P.V.E. McClintock
}

\begin{abstract}
We consider theoretically the spontaneous oscillations of an elastic cylinder in a streaming fluid flow. Such oscillations are responsible for stall flutter in e.g. turbine blades and bridges, or Æolian tones when in the acoustic range. The process of vortex separation from the oscillating surface is modelled as a self-excited oscillator. The vortex separation frequency remains synchronized with the oscillations of the cylinder over a great range of frequency mismatches, enabling the amplitudes and frequencies to be calculated. Only when the vortex separation frequency is much less than the cylinder's natural oscillation frequency does the synchronization break down, and two-frequency oscillations (beats) then occur.
\end{abstract}

\section{Introduction}

It has long been known that, when fluid flows past lengthy bodies, they can radiate Eolian tones [1], sounds resulting from the reaction to the vortex-shedding that creates a Kármán wake [2] downstream of the body, as sketched in Fig. 1. Familiar examples include the "singing" of telegraph wires in the wind, the side movement of an oar in water, and the Eolian harp. The phenomenon also arises in the engineering context as the stall flutter $[3,4]$ of e.g. helicopter rotors, turbine blades, suspension bridges, steel factory pipes, and periscopes of submarines $[5,6,7,8,3,9]$. We will refer to all such self-oscillations as stall flutter, and will not try to distinguish $[8,10]$ between the cases of immovable or flexible cylinders because in both cases there occurs the excitation of self-oscillations. Stall flutter often manifests when one of the body's natural oscillation frequencies is close to the frequency at which vortex separation would occur from the same body while immovable. The rotary oscillations of wires caused by stall flutter are similar in form to thermo-mechanical self-oscillations [11]. Stall flutter is known to have been the underlying cause of many technical disasters of which one of the best known is probably the collapse of the Tacoma Narrows suspension bridge [6, 3]. Despite its prevalence and widespread importance, many experimental results and much numerical analysis $[12,13]$, there is not yet to our knowledge a strict quantitative theory of stall flutter. 


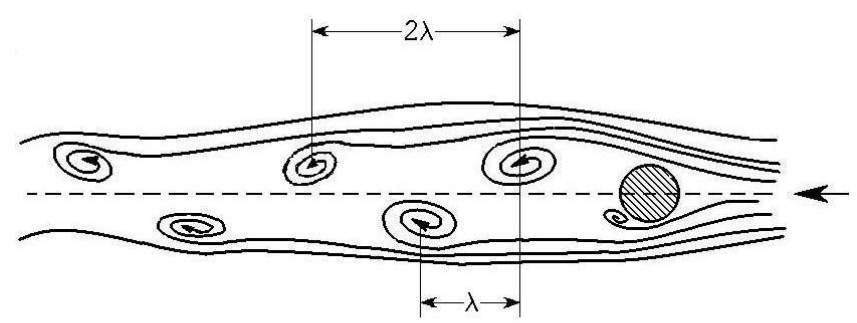

Figure 1. Schematic diagram of the Kármán wake for streamlined flow around a cylinder

Stall flutter is often interpreted as the excitation of resonant oscillations under the action of a periodic force caused by the vortex separation with a frequency dependent on the flow velocity, and the size and shape of the streamlined body. The force frequency may be found from the condition that the Strouhal number St $=f D / U_{0}$ remains approximately constant, where, $f$ is the frequency of vortex separation, $D$ is the cylinder diameter, and $U_{0}$ is the flow velocity. We comment that such explanations are in immediate conflict with the observation that stall flutter of sufficiently long ropes can be maintained by flow at very different velocities over different sections of the rope. In reality, as we shall see below, stall flutter does not correspond simply to forced oscillations. Rather, it is a unified self-oscillatory phenomenon.

Because the process of vortex separation is evidently of a self-oscillatory character $[11,12,14,15,16]$, and the streamlined body represents an oscillatory element with one or several natural frequencies, the stall flutter phenomenon must be similar to self-oscillations in a system containing both active and passive oscillatory elements. In this paper we therefore model stall flutter as an interaction phenomenon arising between a self-excited oscillator (the flow oscillator) $[14,15,16]$ corresponding to the periodic vortex separation, and a passive oscillator (the elastic cylinder around which the fluid flows). Note that the problems of this type were first investigated and solved by physicists of the Mandelstam school in Moscow (including A. Andronov, A. Witt, S. Strelkov and A. Skibarko), in 1934. The corresponding physical effects are known in textbooks on oscillation theory as pulling phenomena.

\section{Stall flutter as a self-oscillatory phenomenon}

Stall flutter is remarkably similar to self-oscillations in a system containing both active and passive oscillatory elements [11]. A classic example of such a system is an oscillator with an additional oscillatory circuit $[17,18,19,20]$. So we may anticipate that all of the well-established features of such systems (e.g. frequency-pulling and the characteristic variation of the oscillation amplitudes as the frequency mismatch varies) will appear in 


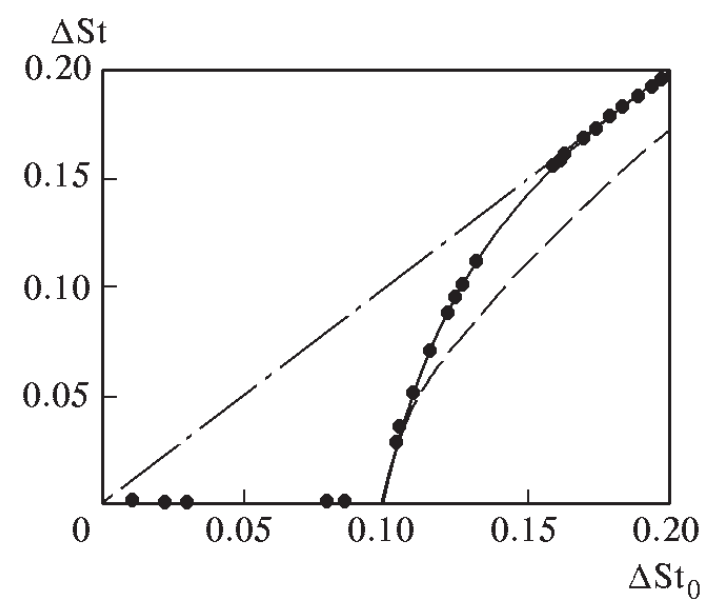

Figure 2. The dependence of the mismatch between the frequency of vortex separation from the oscillated ( $f$ and immovable $f_{0}$ cylinders $\Delta S t=\left(f-f_{0}\right) D / U_{0}$ on the mismatch between the frequency $f_{c}$ of the cylinder oscillations and $f_{0} \Delta \mathrm{St}_{0}=\left(f_{c}-f_{0}\right) D / U_{0}$ expressed in terms of the Strouhal numbers. From experimental data in [25]

the case of stall flutter too. Before attempting to model stall flutter in this way, we make a few general remarks about philosophy of modelling.

\subsection{Levels of modelling}

Modeling stall flutter in the way suggested above does not, of course, signify that such a complex phenomenon is described by equations identical to those of an oscillator with an additional oscillatory circuit. It is clearly evident that these equations are not isomorphic with those for stall flutter. Due to the well-known universality of the laws of oscillation theory, however, we can describe a wide range of oscillation phenomena with relatively simple models described by known equations [21]. For example, turbulent processes in submerged jets can be modeled successfully $[22,23]$ by the equation of a pendulum with a randomly vibrated suspension axis, and the dynamics of the human cardiovascular system can be modeled successfully [24] with Poincaré oscillators. In the present case, we use a generic oscillator to model the observed behaviour of the system, rather than approaching it via the mechanics of continuous media (hydrodynamics). There are numerous other examples in science of high level modeling of a similar kind, where one models an observed phenomenon rather than trying to predict it from a first-principles approach based on the properties of more fundamental elements (e.g. molecules or continua or cells) of the system.

Note that, in contradistinction to some of the earlier work on the oscillator with an additional oscillatory circuit $[17,18,19,20]$, where only one-frequency regime was considered, we show below that in our system a two-frequency regime exists for a small range of mismatches between the vortex separation and cylinder oscillation frequencies. 


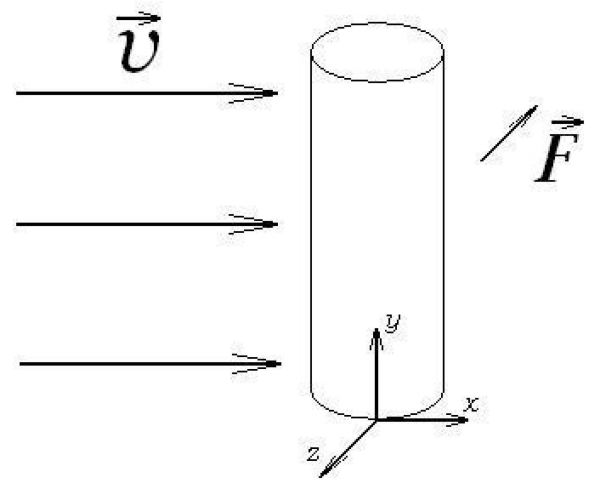

Figure 3. Schematic image of the oscillations of an elastic cylinder of length $l$ taking place along axis $z$ in the case of flowing in the direction of axis $x$

\subsection{Vortex separation as self-oscillations. Synchronization of the vortex separation} process

To justify the self-oscillatory character of the vortex separation process, we note first that the velocity and pressure pulsations at any point behind a streamlined body have a rather narrow spectrum with a well-defined maximum. Detemple-Laake and Eckelmann have already shown [14] experimentally that the vortex separation process from a streamlined body behaves like a nonlinear oscillator whose frequency can be pulled by up to $25 \%$ by acoustic forcing. Secondly, this process from a streamlined body may be synchronized by oscillations of the body at a frequency that is approximately divisible by the frequency of vortex separation from the same body when immovable $[1,26,27,28,25]$. Thirdly, the dependence of the vortex separation from the oscillating body on the mismatch between the body oscillation frequency $f_{b}$ and the frequency of vortex separation from the immovable body is very similar to the well-known dependence of the frequency of synchronized oscillations of a van der Pol oscillator on the mismatch between the free self-oscillation frequency and the external forcing frequency [21]: see e.g. Fig. 2 constructed from the experimental data of [25]. In this figure $\Delta \mathrm{St}=\left(f-f_{0}\right) D / U_{0}$ and $\Delta \mathrm{St}_{0}=\left(f_{\mathrm{c}}-f_{0}\right) D / U_{0}$ are the mismatches between the frequencies of vortex separation from the oscillating $f$ and the immovable $f_{0}$ cylinders expressed in terms of the Strouhal numbers, $f_{c}$ is the frequency of the cylinder oscillations, $D$ is the cylinder diameter, and $U_{0}$ is the flow velocity. For comparison the dependence $\Delta \mathrm{St}=\sqrt{\left(\Delta \mathrm{St}_{0}\right)^{2}-\left(\Delta \mathrm{St}_{0}\right)_{\mathrm{s}}^{2}}$, where $\left(\Delta \mathrm{St}_{0}\right)_{\mathrm{s}}$ is the half-width of the synchronization region, is shown in the same figure by a dashed line.

This dependence should be valid in the case of synchronization of the oscillator by a small harmonic external force. However, judging by the fact that the synchronization region is rather wide, the amplitude of the cylinder oscillations was significant, resulting in a steeper dependence of $\Delta \mathrm{St}$ on $\Delta \mathrm{St}_{0}$. As in other oscillators synchronization can occur, not only for the fundamental frequency, but also for its harmonics and subharmonics [27]. 


\section{A mathematical model of stall flutter}

We therefore consider the oscillations of an elastic circular cylinder of length $l$ with fixed ends, placed transversely to the flow as shown in Fig. 3. The streamlines around a circular cylinder have been considered in many books [29, 9] and papers [27, 30]. A Kármán vortex wake [31] is formed behind a streamlined cylinder. For the Reynolds numbers $40<\operatorname{Re}<150$ it is regular, and for $150<\operatorname{Re}<300$ it is turbulent. For $300<\operatorname{Re}<2 \cdot 10^{5}$ the Kármán wake becomes again close to regular, but with turbulent bursts. After this, for $\operatorname{Re}>5 \cdot 10^{6}$, a dominant vortex separation frequency is again observed in the wake spectrum [32]. It should be emphasized that such an alternation between regions of different behavior as a parameter changes is typical of chaotic selfoscillatory systems $[33,11]$. In particular, the region $300<\operatorname{Re}<2 \cdot 10^{5}$ may be considered as a region of intermittency. The scenario represents additional evidence that the formation of the Kármán wake is a self-oscillation process. In what follows we will consider only that range of Reynolds numbers where vortex separation occurs in an almost periodic manner.

A stalling streamline in the direction of the $x$ axis with velocity $V$ (identical for all cylinder sections [34]) results in a nearly periodic lift force $F_{k z}(t)$ along the $z$ axis, and a drag force $F_{r x}(t)$ along the $x$ axis toward the flow. The latter is made up of: a slowly-changing component; and a component that is nearly periodic at half the period of the lift force.

For an immovable cylinder, over a wide range of the Reynolds numbers, the frequency of the lift force is equal to that of vortex separation $\omega_{v}$, and the frequency of the drag force is equal to $2 \omega_{v}$ [27]. The difference arises because the lift force frequency is defined by the distance between vortices along one side of the Kármán wake $(2 \lambda)$, whereas the drag force frequency is defined by the distance between vortices along both sides of the Kármán wake $(\lambda)$ (see Fig. 1).

The frequency $\omega_{v}$ is connected to the Strouhal number St and the flow velocity $V$ by $\omega_{v}=2 \pi \mathrm{St} / D V$, where $D$ is the cylinder diameter. Experiments [9] show that, for $40<\operatorname{Re}<150$, the Strouhal number increases with $\operatorname{Re}$, then becomes approximately constant and equal to 0.2, and then increases again as shown in Fig. 4 [9]. For $2 \cdot 10^{5}<\operatorname{Re}<5 \cdot 10^{6}$ the vortex wake is strongly turbulent, so that the Strouhal number cannot be determined. For $\operatorname{Re}>5 \cdot 10^{6}$, the Strouhal number St $\approx 0.3$.

As far as we are aware, no equations have been proposed that would enable us to describe rigorously the lift and drag forces for a stalling streamline. However, expressions for their amplitudes and frequencies have been deduced via dimensional considerations $[29,27,30,3,9]$. The amplitudes of the lift and drag forces $F_{k z}(t)$ and $F_{r x}(t)$ are correspondingly

$$
A_{k z}=c_{k}(\operatorname{Re}) S_{y z} \frac{\rho V^{2}}{2}, \quad A_{r x}=c_{r}(\operatorname{Re}) S_{y z} \frac{\rho V^{2}}{2},
$$

where $c_{k}(\operatorname{Re})$ and $c_{r}(\mathrm{Re})$ are factors depending on the quality of streamlining (the worse the streamlining, the larger the values of $c_{k}$ and $c_{r}$ ) and on the Reynolds number, $S_{y z}$ is 
the area of the body projection on the plane $y z$ normal to the flow direction. We note that the factor $c_{r}(\mathrm{Re})$ is always much less than $c_{k}(\mathrm{Re})$, i.e. the oscillating component of the drag force is small in comparison to the lift force.

For an oscillating cylinder, forces arising from the non-stationarity of the streamline must be added to the forces $F_{k z}(t)$ and $F_{r x}(t)$ defining the added hydrodynamic mass and an additional damping factor; and the factors $c_{k}(\operatorname{Re})$ and $c_{r}(\operatorname{Re})$ will depend on the oscillation amplitude.

Considering the oscillating cylinder as a stretched string, we write the equations of its oscillations along axes $x$ and $z$ as

$$
\begin{aligned}
& \rho_{s} \frac{\partial^{2} u_{x}}{\partial t^{2}}+\beta \frac{\partial u_{x}}{\partial t}-T \frac{\partial^{2} u_{x}}{\partial y^{2}}=\frac{F_{r x}(t)}{A_{r x}} \\
& \rho_{s} \frac{\partial^{2} u_{z}}{\partial t^{2}}+\beta \frac{\partial u_{z}}{\partial t}-T \frac{\partial^{2} u_{z}}{\partial y^{2}}=\frac{F_{k z}(t)}{A_{k z}}
\end{aligned}
$$

where $u_{x}(y, t) / A_{r x}$ and $u_{z}(y, t) / A_{k z}$ are the relative cylinder displacements along $x$ and $z$, $\rho_{s}$ is the linear density of the string, $T$ is the string tension, and $\beta$ is the damping factor. If the cylinder ends are fixed, the boundary conditions are $u_{x}(0, t)=0, \quad u_{x}(l, t)=$ $0, \quad u_{z}(0, t)=0, \quad u_{z}(l, t)=0$.

Noting that $F_{r x}(t)$ and $F_{k z}(t)$ are nonzero only for $0 \leq y \leq l$, and recalling that the drag force frequency is twice the lift force frequency, we write

$$
\begin{aligned}
& F_{r x}(t)=A_{r x} \sum_{j=1}^{\infty} f_{r x}^{(j)}(t) \sin \left(\frac{2(2 j-1) \pi}{l} y\right), \\
& F_{k z}(t)=A_{k z} \sum_{j=1}^{\infty} f_{k z}^{(j)}(t) \sin \left(\frac{(2 j-1) \pi}{l} y\right),
\end{aligned}
$$

where

$$
f_{r x}^{(j)}(t)=\frac{F_{r x}(t)}{2(2 j-1) \pi A_{r x}}, \quad f_{k z}^{(j)}(t)=\frac{F_{k z}(t)}{(2 j-1) \pi A_{k z}} .
$$

If the frequency of vortex separation from the oscillating cylinder is close to its $n$-th

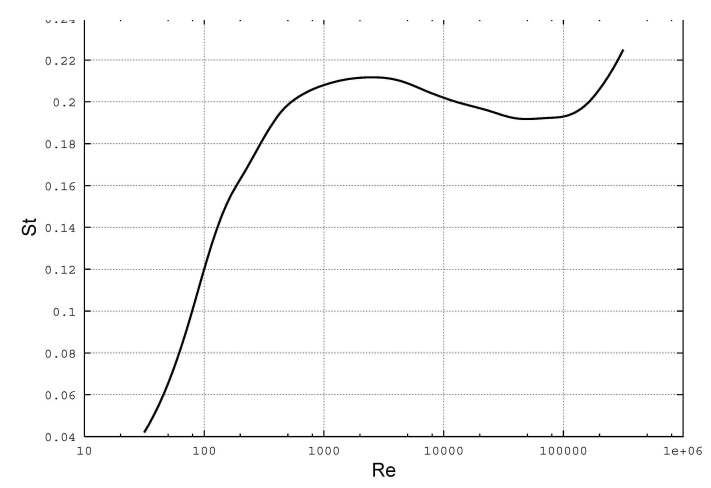

Figure 4. The dependence of the Strouhal number on the Reynolds number for transversal streamline of an immovable circular cylinder 
natural frequency then we can retain only one term in each of the expansions (3), i.e.

$$
\begin{aligned}
& F_{k z}(t)=A_{k z} f_{k z}^{(n)}(t) \sin \left(\frac{(2 j-1) \pi}{l} y\right), \\
& F_{r x}(t)=A_{r x} f_{r x}^{(2 n)}(t) \sin \left(\frac{2(2 j-1) \pi}{l} y\right) .
\end{aligned}
$$

Solving Eqs. (2) by expansion in terms of the natural functions for an undamped string with fixed ends, and retaining only the first term in the expansion, we have

$$
\begin{aligned}
& u_{x}(y, t)=U_{x}^{(j)}(t) \sin \left(\frac{2(2 j-1) \pi}{l} y\right), \\
& u_{z}(y, t)=U_{z}^{(j)}(t) \sin \left(\frac{(2 j-1) \pi}{l} y\right) .
\end{aligned}
$$

In so doing we find for functions $U_{z}^{(n)}(t)$ and $U_{x}^{(2 n)}(t)$ the following equations:

$$
\begin{aligned}
& \frac{d^{2} U_{z}}{d t^{2}}+2 \delta \frac{d U_{z}}{d t}+\omega_{n}^{2} U_{z}=\frac{f_{k z}^{(n)}(t)}{\rho_{s}}, \\
& \frac{d^{2} U_{x}}{d t^{2}}+2 \delta \frac{d U_{x}}{d t}+4 \omega_{n}^{2} U_{x}=\frac{f_{r x}^{(2 n)}(t)}{\rho_{s}},
\end{aligned}
$$

where $\delta=\beta /\left(2 \rho_{s}\right) \ll \omega_{n}$.

Owing to the difference of the oscillation frequencies in the $x$ and $z$ directions, the resultant oscillations will in general be of complex form. Moreover, in the ranges of Reynolds numbers where the vortex separation process is random, these oscillations will also be random. Precisely this feature was observed prior to collapse of the Tacoma bridge, and it also appears in the stall flutter of power lines.

As indicated above, the vortex separation process is self-oscillatory in nature. It arises owing to the formation behind the streamlined body of a backwash, providing the feedback needed for excitation of the self-oscillations. To calculate the forces $F_{k z}(y, t)$ and $F_{r x}(y, t)$ we take the van der Pol oscillator as a model of the excitation of self-oscillations phenomenon [11]. Taking account of the experimental fact that the cylinder oscillations can synchronize the vortex separation, we write the following model equations for $f_{r x}(t)$ and $f_{k z}(t)$ :

$$
\begin{aligned}
& \frac{d^{2} f_{k z}}{d t^{2}}-\mu\left(1-4 f_{k z}^{2}\right) \frac{d f_{k z}}{d t}+\omega_{v}^{2} f_{k z}=m_{1} U_{z}, \\
& \frac{d^{2} f_{r x}}{d t^{2}}-\mu\left(1-4 f_{r x}^{2}\right) \frac{d f_{r x}}{d t}+4 \omega_{v}^{2} f_{r x}=m_{2} U_{x},
\end{aligned}
$$

where $\mu$ is a small parameter, and the coupling coefficients $m_{1,2}$ are assumed small. The terms $m_{1} U_{z}$ and $m_{2} U_{x}$ are responsible for the synchronization of vortex separation by the cylinder oscillations. Note that $\mu$ represents a negative friction that is inversely proportional to the transition time of the self-oscillations. Depending on the value of $\mu$, therefore, the oscillations can in principle occur at almost any frequency and are not confined to the acoustic range. 

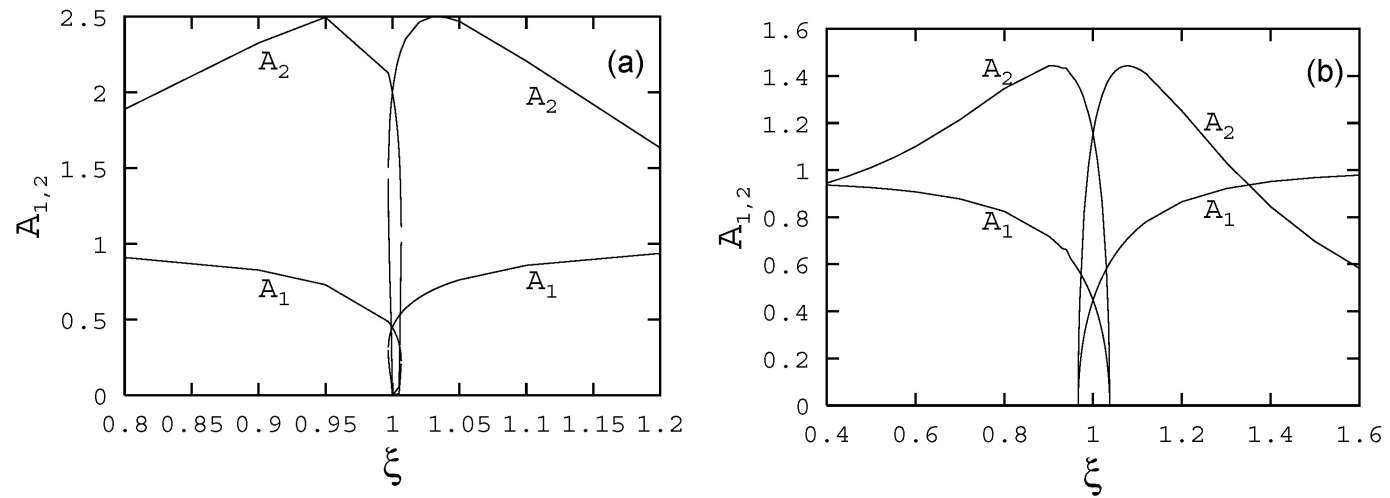

Figure 5. The dependences of the self-oscillation amplitudes of the lift force $\left(A_{1}\right)$ and cylinder $\left(A_{2}\right)$ on the frequency mismatch $\xi$ for $\delta / \omega_{n}=0.1$ with (a) $m_{1} / \omega_{n}^{2}=0.05$, $m_{2} / \omega_{n}^{2}=1$ and (b) $m_{1} / \omega_{n}^{2}=0.15, m_{2} / \omega_{n}^{2}=1$

It follows from Eqs. (6) that, for $U_{x}(t) \equiv U_{z}(t) \equiv 0$, the stationary values of $F_{k z}(t)$ and $F_{r x}(t)$ are

$$
\begin{aligned}
& F_{k z}(t)=A_{k z} f_{k z}(t) \approx A_{k z} \cos \left(\omega_{v} t\right), \\
& F_{r x}(t)=A_{r x} f_{r x}(t) \approx A_{r x} \cos \left(2 \omega_{v} t+\varphi\right),
\end{aligned}
$$

where $\varphi$ represents the phase shift between the drag and lift forces. Note that (7) are in full accord with the expressions for the lift and drag forces given in [27].

Eqs. (5) and (6) describe two independent self-oscillatory systems each of which has two degrees of freedom. In terms of oscillation theory, each of them is an oscillator with an additional oscillatory circuit coupled to the main one by a capacitance [19]. Due to oscillations of the instantaneous streamline velocity as the cylinder oscillates, Eqs. (6) could be nonlinearly coupled, but we neglect this possibility. A similar oscillator, but with inductive coupling, was studied by Andronov and Witt with use of the Poincaré small parameter method [17], and by Strelkov using qualitative methods [18]. These works considered a single-frequency approximation, i.e. the synchronous regime. In each case, parameter ranges were identified where frequency-pulling and quenching of the self-oscillations occurred. It should be noted that the character of coupling may be defined only by using experimental data. We have set capacitive coupling because it results in new phenomenon (the beat regime) in comparison with inductive one.

We now consider the system described by the first equations in (5) and (6). For an approximate solution we apply the averaging method of Bogolyubov [35] as developed by Mitropol'sky [36], but in the form described in [20]. It should be noted that a model described by the equations similar to $(5),(6)$ was considered only in one-frequency regime by another method in [16]. Rewriting Eqs. (5), (6) with a conditional small parameter $\epsilon$ and dimensionless time $\tau=\omega t$, where $\omega$ is the self-oscillation frequency,

$$
\ddot{f}_{k z}+f_{k z}=\epsilon\left[\left(1-\frac{\xi^{2}}{\tilde{\omega}^{2}}\right) f_{k z}+\frac{\tilde{m}_{1}}{\tilde{\omega}^{2}} U_{z}+\frac{\tilde{\mu}}{\tilde{\omega}}\left(1-4 f_{k z}^{2}\right) \dot{f}_{k z}\right]
$$



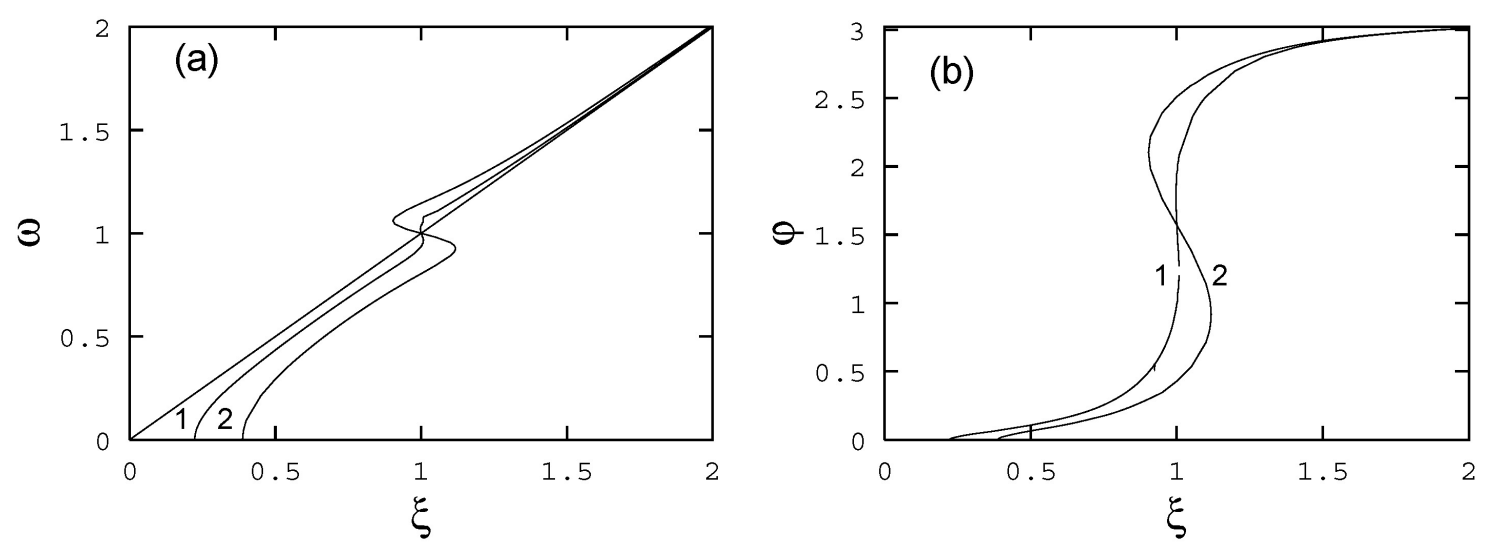

Figure 6. The dependences of relative self-oscillation frequency $(a)$ and phase difference between oscillatory circuits 1 and $2(b)$ on the frequency mismatch for $\delta / \omega_{2}=0.1: m_{1} / \omega_{n}^{2}=0.05, m_{2} / \omega_{n}^{2}=1$ (curves 1$) ; m_{1} / \omega_{n}^{2}=0.15, m_{2} / \omega_{n}^{2}=1$ (curves 2)

$$
\ddot{U}_{z}+U_{z}=\epsilon\left[\frac{f_{k z}(t)}{\rho_{s} \omega_{n}^{2} \tilde{\omega}^{2}}+\left(1-\frac{1}{\tilde{\omega}^{2}}\right) U_{z}-\frac{2 \tilde{\delta}}{\tilde{\omega}} \dot{U_{z}}\right] .
$$

Here $\tilde{\mu}=\mu / \omega_{n}, \tilde{\delta}=\delta / \omega_{n}, \tilde{m}_{1}=m_{1} / \omega_{n}^{2}, \xi=\omega_{v} / \omega_{n}$ is the mismatch between the frequencies of vortex separation $\left(\omega_{v}\right)$ and cylinder oscillation $\left(\omega_{n}\right), \tilde{\omega}=\omega / \omega_{n}$ is the relative self-oscillation frequency, and dots imply differentiation with respect to dimensionless time $\tau$.

We now introduce new variables $A_{1,2}(\tau)$ and $\Phi_{1,2}(\tau) \equiv \tau+\varphi_{1,2}(\tau)$ defined by the equations

$$
\begin{array}{ll}
f_{k z}(\tau)=A_{1}(\tau) \cos \Phi_{1}(\tau), & \dot{f}_{k z}(\tau)=-A_{1}(\tau) \sin \Phi_{1}(\tau), \\
U_{z}(\tau)=A_{2}(\tau) \cos \Phi_{2}(\tau), & \dot{U}_{z}(\tau)=-A_{2}(\tau) \sin \Phi_{2}(\tau) .
\end{array}
$$

In view of (9), Eqs. (8) become

$$
\begin{aligned}
& \dot{A}_{1} \cos \Phi_{1}-A_{1} \dot{\varphi}_{1} \sin \Phi_{1}=0, \quad-\left(\dot{A}_{1} \sin \Phi_{1}+A_{1} \dot{\varphi}_{1} \cos \Phi_{1}\right)= \\
& \epsilon\left[\left(1-\frac{\xi^{2}}{\tilde{\omega}^{2}}\right) A_{1} \cos \Phi_{1}-\frac{\tilde{\mu}}{\tilde{\omega}}\left(1-4 A_{1}^{2} \cos ^{2} \Phi_{1}\right) A_{1} \sin \Phi_{1}+\frac{\tilde{m}_{1}}{\tilde{\omega}^{2}} A_{2} \cos \Phi_{2}\right], \\
& \dot{A}_{2} \cos \Phi_{2}-A_{2} \dot{\varphi}_{2} \sin \Phi_{2}=0, \quad-\left(\dot{A}_{2} \sin \Phi_{2}+A_{2} \dot{\varphi}_{2} \cos \Phi_{2}\right)= \\
& \epsilon\left[\left(1-\frac{1}{\tilde{\omega}^{2}}\right) A_{2} \cos \Phi_{2}+\frac{2 \tilde{\delta}}{\tilde{\omega}} A_{2} \sin \Phi_{2}+\frac{\tilde{m}_{2}}{\tilde{\omega}^{2}} A_{1} \cos \Phi_{1}\right]
\end{aligned}
$$

where $\tilde{m}_{2}=1 /\left(\rho_{s} \omega_{n}^{2}\right)$. It should be noted that Eqs. (10) are exact equations for the amplitudes and phases.

Proceeding to apply the averaging method, in the first approximation it is necessary to solve Eqs. (10) relative to $\dot{A}_{1}, \dot{A}_{2}, \dot{\varphi}_{1}, \dot{\varphi}_{2}$ and to average the equations found over the 
fast time $\tau$. We thus obtain:

$$
\begin{aligned}
\dot{A}_{1}= & \frac{\epsilon}{2 \tilde{\omega}}\left(\tilde{\mu}\left(1-A_{1}^{2}\right) A_{1}-\frac{\tilde{m}_{1}}{\tilde{\omega}} A_{2} \sin \varphi\right), \\
\dot{A}_{2} & =-\frac{\epsilon}{2 \tilde{\omega}}\left(2 \tilde{\delta} A_{2}-\frac{\tilde{m}_{2}}{\tilde{\omega}} A_{1} \sin \varphi\right), \\
\dot{\varphi}_{1} & =-\frac{\epsilon}{2 A_{1}}\left[\left(1-\frac{\xi^{2}}{\tilde{\omega}^{2}}\right) A_{1}+\frac{\tilde{m}_{1}}{\tilde{\omega}^{2}} A_{2} \cos \varphi\right], \\
\dot{\varphi}_{2} & =-\frac{\epsilon}{2 A_{2}}\left[\left(1-\frac{1}{\tilde{\omega}^{2}}\right) A_{2}+\frac{\tilde{m}_{2}}{\tilde{\omega}^{2}} A_{1} \cos \varphi\right],
\end{aligned}
$$

where $\varphi=\varphi_{1}-\varphi_{2}$ is the phase difference between the lift force oscillations and cylinder oscillations.

In the steady-state regime all derivatives in Eqs. (10), (11) may be set equal to zero, yielding the following equations for the amplitudes $A_{1}, A_{2}$, phase difference $\varphi$ and relative self-oscillation frequency $\tilde{\omega}$ :

$$
\begin{aligned}
& \tilde{\mu}\left(1-A_{1}^{2}\right)-\frac{\tilde{m}_{1}}{\tilde{\omega}} \frac{A_{2}}{A_{1}} \sin \varphi=0, \quad 2 \tilde{\delta} \frac{A_{2}}{A_{1}}-\frac{\tilde{m}_{2}}{\tilde{\omega}} \sin \varphi=0, \\
& 1-\frac{\xi^{2}}{\tilde{\omega}^{2}}+\frac{\tilde{m}_{1}}{\tilde{\omega}^{2}} \frac{A_{2}}{A_{1}} \cos \varphi=0, \quad\left(1-\frac{1}{\tilde{\omega}^{2}}\right) \frac{A_{2}}{A_{1}}+\frac{\tilde{m}_{2}}{\tilde{\omega}^{2}} \cos \varphi=0 .
\end{aligned}
$$

Eliminating the amplitude ratio $A_{2} / A_{1}$ from Eqs. (12, (12), we find the following equations for the phase difference $\varphi$ and the relative frequency $\tilde{\omega}$ :

$$
\tilde{\omega}\left(\tilde{\omega}^{2}-\xi^{2}\right)+\frac{\tilde{m}_{1} \tilde{m}_{2}}{4 \tilde{\delta}} \sin 2 \varphi=0, \quad\left(\tilde{\omega}^{2}-1\right) \tan \varphi+2 \tilde{\delta} \tilde{\omega}=0
$$

Next, we calculate the dependence of the self-oscillation amplitudes on the frequency mismatch. From (12), (12),

$$
A_{1}=\sqrt{1-\frac{\tilde{m}_{1} \tilde{m}_{2}}{2 \tilde{\mu} \tilde{\delta} \tilde{\omega}^{2}} \sin ^{2} \varphi}, \quad A_{2}=\frac{\tilde{m}_{2}}{2 \tilde{\delta} \tilde{\omega}} A_{1} \sin \varphi .
$$

Substituting $\tilde{\omega}(\xi)$ and $\varphi(\xi)$ calculated above, we find the dependences of $A_{1}$ and $A_{2}$ on $\xi$. Some examples are shown in Fig. 5.

\section{Discussion and conclusions}

The dependences of the lift force and cylinder oscillation amplitudes $\left(A_{1}\right.$ and $\left.A_{2}\right)$ are quite different from each other, although each has a minimum at $\xi=1$. On either side of the minimum, the oscillation amplitude of the lift force increases monotonically, whereas that of the cylinder exhibits maxima.

Examples of the dependences of $\omega / \omega_{n}$ and $\varphi$ on the mismatch $\xi$ are shown in Fig. 6. It is seen from Fig. 6(a) that, for mismatches $\xi \leq \xi_{\text {cr }}$, i.e. for comparatively small frequencies of vortex separation in the case of an immovable cylinder, synchronous selfoscillations are impossible. The value of $\xi_{\text {cr }}$ can be found analytically. It is equal to $\xi_{\mathrm{cr}}=\sqrt{m_{1} m_{2} / \omega_{n}^{4}}$ 
As the mismatch increases, the self-oscillation frequency tends to the frequency of the oscillator without its additional circuit, but not monotonically. In the vicinity of $\xi=1$, frequency-pulling occurs when the self-oscillation frequency depends, not only on the initial value of the frequency, but also on the direction of its variation. In this region, our results coincide with those reported earlier [19]. The dependences in Figs. 5 and 6 are calculated without consideration for the stability of the solutions, but these are readily found from Eqs. (5), (6). Unsurprisingly, some parts of the dependences near $\xi=1$ in these figures turn out to be unphysical and drop out.

The reason that the results shown in Figs. 5 and 6 differ from the corresponding dependences found in [16] is probably associated with the different methods used in the respective calculations. Facchinetti et al [16] simply equated the coefficients of the main harmonics whereas, here, we have applied well-established methods of oscillation theory. Our results are consistent with those of Teodorchik [19] obtained by his energetic method.

In summary, we have proposed a theory of stall flutter, modeled as a self-excited oscillator with an additive oscillatory circuit. We have shown that this process is accompanied by synchronization and pulling. It has enabled us to calculate the amplitudes and frequencies of the resultant self-oscillations. We have found that vortex separation may be synchronized by the natural oscillations of the cylinder, and that there should also exist a regime of beating for sufficiently large frequency mismatch. Such effects would be consistent with the frequency-pulling observed earlier [14] under acoustic forcing, and they invite experimental investigation.

We emphasize that our present theory allows us to find only the regular regimes of stall flutter. But we infer that the model described by Eqs. (4), (5), plus some additive nonlinear terms, will describe the chaotic regimes as well. As indicated above, such regimes are potentially damaging for engineering constructions.

\section{Acknowledgements}

The work was supported by the Engineering and Physical Sciences Research Council and by the Royal Society (London) whom PSL gratefully acknowledges for the award of a visiting research fellowship.

\section{References}

[1] Richardson E G 1923 Proc. Phys. Soc. (Lond.) 36 153-167

[2] von Kármán T 1911 Göttingen Nachrichten 509-517

[3] Panovko Y G and Gubanova I I 1965 Stability and Oscillations of Elastic Systems (New York: Consultants Bureau)

[4] Note the distinction between stall flutter associated with vortex shedding, and the classical flutter arising from coupling between two or more degrees of freedom. The excitation of classical flutter requires $[5,17]$ higher flow velocities than stall flutter 
[5] Goldenblat I I 1947 Contemporary Problems of Vibration and Stability of Engineering Constructions (in Russian) (Moscow: Gosstroyizdat)

[6] Rocard Y 1949 Dynamique Générale des Vibrations (in French) (Paris: Masson)

[7] Halfman R L, Johnson H C and Haley S M 1951 Evaluation of High-Angle-of-Attack AerodynamicDerivative Data and Stall-Flutter Prediction Techniques Report. No. A712083 (Washington: NASA)

[8] Bisplinghoff R L, Ashley H and Halfman R L 1955 Aeroelasticity (Cambridge Mass.: AddisonWesley)

[9] Försching H W 1974 Grundlagen der Aeroelastik (Berlin: Springer-Verlag)

[10] Kazakevich M I 1987 Aerodynamics of Bridges (in Russian) (Moscow: Transport)

[11] Landa P S 1997 Nonlinear Oscillations and Waves (Moscow: Nauka-Fizmatlit)

[12] Ericsson L E and Reding J P 1973 J. Aircraft 10 5-13

[13] Marshall J G and Imregun M 1996 J. Fluids and Structures 10 237-267

[14] Detemple-Laake E and Eckelmann H 1989 Experiments in Fluids 7 217-227

[15] Facchinetti M L, de Langre E and Biolley F 2004 Eur. J. Mech. B/Fluids 23 199-208

[16] Facchinetti M L, de Langre E and Biolle F 2004 J. Fluids and Structures 19 123-140

[17] Andronov A A and Witt A A 1934 Zh. Eksp. Teor. Fiz. 4 122-136

[18] Strelkov S P and Skibarko A P 1934 Zh. Eksp. Teor. Fiz. 4 158-171

[19] Teodorchik K F 1952 Self-Oscillatory Systems (Moscow: Gostekhizdat)

[20] Landa P S 1980 Self-Oscillations in Systems with Finite Number of Degrees of Freedom (Moscow: Nauka)

[21] Landa P S 1997 Discrete Dynamics in Nature and Society 1 99-110

[22] Landa P S and Zaikin A A 1996 Phys. Rev. E 54 3535-3544

[23] Landa P S and McClintock P V E 2004 Phys. Reports 397 1-62

[24] Stefanovska A and Bračič M 1999 Contemporary Phys. 40 31-55

[25] Fedyaevskii K K and Blyumina L K 1977 Hydrodynamics of Body Separation Streamline in Russian (Moscow: Mashinostroenie)

[26] Pavlikhina M A and Smirnov L P 1958 Izv. AN SSSR, OTN No. 8, 124-127

[27] Bishop R E D and Hassan A Y 1964 Proc. Roy. Soc. (Lond.) A277 51-75

[28] Blyumina L K and Fedyaevskii K K 1969 Izv. AN SSSR, MZhG 4 118-119

[29] Fung Y C 2008 An Introduction to the Theory of Aeroelasticity (New York: Dover)

[30] Bishop R E D and Hassan A Y 1964 Proc. Roy. Soc. (Lond.) A277 32-50

[31] von Kármán T 1912 Phys. Z. 13 49-59

[32] Roshko A 1961 J. Fluid Mech. 10 345-356

[33] Neimark Y I and Landa P S 1987 Stochastic and Chaotic Oscillations (Dordrecht: Kluwer Academic)

[34] The assumption that all sections of the cylinder are streamlined with the same velocity is not essential. Even when this assumption is invalid, the problem may still solved e.g. by use of Galerkin's method (provided, of course, that the motion does not become chaotic)

[35] Bogolyubov N N 1950 Sbornik Instituta Stroitel'noy Mekhaniki AN USSR No. 14, 9-34

[36] Mitropol'sky Y A 1971 The Averaging Method in Nonlinear Mechanics (Kiev: Naukova Dumka) 